\title{
Influence of bulk dielectric polarization upon partial discharge transients effect of heterogeneous dielectric geometry
}

McAllister, lain Wilson; Crichton, George C.

Published in:

IEEE Transactions on Dielectrics and Electrical Insulation

Link to article, DOI:

$10.1109 / 94.839350$

Publication date:

2000

Document Version

Publisher's PDF, also known as Version of record

Link back to DTU Orbit

Citation (APA):

McAllister, I. W., \& Crichton, G. C. (2000). Influence of bulk dielectric polarization upon partial discharge transients effect of heterogeneous dielectric geometry. IEEE Transactions on Dielectrics and Electrical Insulation, 7(1), 124-132. https://doi.org/10.1109/94.839350

\section{General rights}

Copyright and moral rights for the publications made accessible in the public portal are retained by the authors and/or other copyright owners and it is a condition of accessing publications that users recognise and abide by the legal requirements associated with these rights.

- Users may download and print one copy of any publication from the public portal for the purpose of private study or research.

- You may not further distribute the material or use it for any profit-making activity or commercial gain

- You may freely distribute the URL identifying the publication in the public portal 


\title{
Influence of Bulk Dielectric Polarization upon Partial Discharge Transients
}

\section{Effect of Heterogeneous Dielectric Geometry}

\author{
I. W. McAllister and G. C. Crichton \\ Department of Electric Power Engineering, Tochnieal Uniryetsity of Denmatk, Lyngby, Donthark
}

\begin{abstract}
A physically valid theory of partial discharge (PD) transients is based upon the concept of the charge induced upon the detecting electrode by the PD. This induced charge consists of two components. One is associated with the actual space charge in the void, while the other is related to changes in the polarization of the bulk dielectric. These changes are brought about by the ficld produced by the space charge. The magnitude of the induced charge and its components are examined for several heterogencous dielectric systems. It is demonstrated that, in relation to a homogeneous diolectric system, the magnitude of the induced charge either increases of decreases depending on the ratio of the diclectric permittivities and within which dielectric the void is located. It is shown that this behavior is directly related to the magnitude and polarity of the polarization component of the induced charge. Furthermore, we demonstrate that the geometry of the dietectric system and the physical dimensions of the different dielectrics influence in a similar manner the magnitude of the induced charge, although to a lesser degree.
\end{abstract}

\section{INTRODUCTION}

$\mathrm{T}$ THE occurrence of partial discharge (ID) in a gaseous woid leads not only to charge being induced on the detecting electrode, but atso to a change in the polarization $\delta \vec{P}$ of the bulk dielectric. P'edersen et at. have shown that this change influences the magnitude of the charge induced upon the detecting electrode $[1,2]$.

The charge induced on a detecting electrode can be evaluated using either the $\lambda$ function, or the $\phi$ function $[1,2]$. These functions take account of the dielectric polarization either implicitly $(\lambda)$ or explicitly $(\phi)$. Thus by using the two functions it becomes possible to identify the influence which changes in dielectric polarization have upon induced charge. Changes in polarization $\delta \vec{P}$ are related directly to clanges in dectric field $\delta \vec{E}$. In I'D, the source of $\delta \vec{E}$ is the PD space charge which is confined to voids located within the bulk dielectric.

In the present paper, the influence of the system geometry toon $\vec{B} \vec{P}$ is examined for a two-layer diclectric system, It is shown that the component of the induced charge due to $\delta \vec{P}$ may increase or decrease depending upon the ratio of the dielectric permittivities and within which dielectric the void is located. The magnitude of this increase/cecrease is also dependent upon both the dimensions of fhe different dielectrics and the non-uniformity of the system geometry. As such behavior is succinctly described in terms of the Poissonian induced charge [3], we will first introduce this concept.

\section{POISSONIAN INDUCED CHARGE}

Induced charge can be described in terms of either a Poissoniant or a Laplacian component [3]. The laplacian induced charge, which is cqual in magnitude but opposite in polarity to the l'oissonian component, is associated with the change in potential of the detecting clectrode. Consequently, the Laplacian charge is the source of any PD transient sigral.

The Poissonian induced charge is that component of the induced charge which is rigidly linked to the space charge source, and which together with this source gives rise to the 'basic Poisson field' [2]. This field is gonerated by the $\mathrm{PD}$.

Mathematically, the firlal value of the Poissonian indured charge of due to a pD can be expressed as the sum of two components

$$
q=q_{t}+q_{p}
$$


where $a_{\nu}$ is the induced charge directly associated with the space charge in the void, and $q_{p}$. represents the induced charge related to the change in dielectric polarization $\delta \vec{P}$ due to the presence of this space charge [2]. With seference to the detecting electrode and the induced charge, the effect of the void wall charges can be cquated to the effect of an electric dipole of moment $\vec{p}$ located within the void [1].

The total Poissonian induced charge arising from a dipole is given by

$$
q=-\ddot{j} \cdot \ddot{\nabla} \lambda
$$

where $\lambda$ represents the proportionality factor between the charge in the void and the induced charge on the detecting clectrode. The $\lambda$ function is a solution of the general Iaplace equation [2]

$$
\vec{\nabla} \cdot(\varepsilon \vec{\nabla} \lambda)=0
$$

in which $\varepsilon$ denotes permittivity.

The component of the Poissonian intuced charge related to the void space charge alone may be obtained via another proportionality factor b; viz.

$$
\eta_{\mu}=-\vec{\mu} \cdot \vec{\nabla} \phi
$$

where the $\phi$ function is a solution of the reduced Laplaces sequation [2]

$$
\nabla^{2} \phi=0
$$

Hence, from (1), (2) and (4), the polarization component $q_{1}$, of the Ioissomian induced charge may be expressed as

$$
q_{p^{2}}=-\vec{\mu} \cdot(\vec{\nabla} \lambda-\vec{\nabla} \phi)
$$

\section{THE $\lambda_{0}$ FUNCTION}

If the dimensions of the void are such that $\vec{\nabla} \lambda$ may be assumed constant within the void, then we can introduce another function $\lambda$. which represents the umperturbed $\lambda$ function. That is, the system is considered woid free. $\Lambda$ s $\lambda$ is a solution of Laplaces equation, then by mathematical analogy with electrostatic ficlds, the relationship between the $\lambda$ and $\lambda_{0}$ functions is given by

$$
\vec{\nabla} \lambda a: h \vec{\nabla} \lambda_{0}
$$

For the type of void under consideration, the parameter $t$ will be a scalar which depends on the void geonctry and the reative permittivity of the associated bulk medium. Following the introduction of $\lambda_{0}$, the total Poissonian induced charge on the detecting chectrode may be expressed as

$$
q=i-h_{\vec{p}} \cdot \vec{\nabla} \lambda_{0}
$$

Furthermore, using (6) and (7) we rc-express $q_{p}$ as

$$
q_{p}=-\overrightarrow{p l} \cdot\left(h \vec{\nabla} \lambda_{t\}}-\vec{\nabla} \phi\right)
$$

For a homogeneous bulk dielectric system, $\lambda_{0}$ is a solution of the reduced Laplace equation, and henee in such siftations $\lambda_{11}$ and $\phi$ are synonymous. As a consequenoe (9) reduces to

$$
q_{p}=-(h-1) \vec{h} \cdot \vec{\nabla} \lambda_{0}
$$

Combining $(8)$ and $(10)$, we arrive at the expression

$$
\frac{q}{q}=: \frac{h-1}{h}
$$

For such a homogencous situation, the eflect of void geometry upon the behavior of $q_{p}$ has been reported; see [2].
Th practice howewer, nearly all equipment will consist of a heterugeneous dielectric system. Nevertheless for a very restricted class of dielectric gromotries, e.g. a conxial disc spacer, $\lambda_{0}$ remains a solution of the reduced Laplace equation, and thus $\lambda_{0}$ and $\phi$ are again synonymous.

To examine the sittation in which $\lambda_{0}$ is a solution of the general Laplace cluation, we consider several specific hoterogeneous dielectric systoms as a means of cxamining the behivior of $u_{p}$. Towever, as indiated by (9), three independent vectors are involved in the evaluntion of $q_{p}$. Hence, for an initial investigation of this behavior, we will limit our study to situations for which the three vectors are parallel.
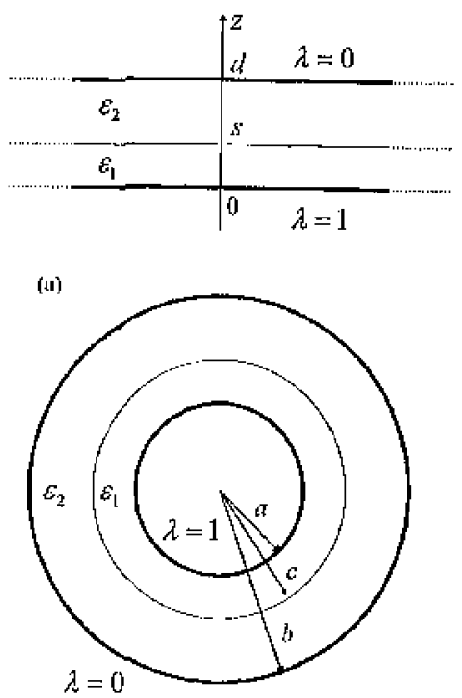

$$
\text { (II) }
$$

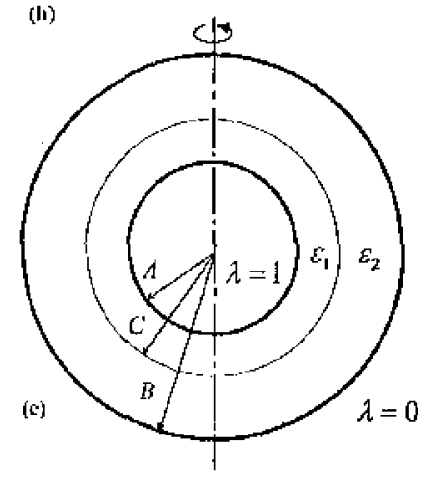

Figure 1. The hetetogenous bulk dielectric systems, (a) planar geometry, (b) cylindrical geomery, (c) spherical gemetry.

Three simple geometrics which fulfill this reguirement are shown in Figure 1 ; namely a planar, a cylindrical and a sphorical geometry. Each supports a two layer diclectric system. Because the derivation of the recovant $\vec{\nabla} \lambda_{0}$ and $\vec{\nabla} \phi$ functions is straightforward, this is confined to the $A$ ppendix. It should be noted that $\lambda=1$ denotes the detecting electrode. 


\section{POISSONIAN INDUCED CHARGE}

To undertake a comparative assessment of the influence of the diclectric geometry upon PD transients we will assume that the dipole $i t_{t}$ associated with the charge which accumulates at the void wall following a $P D_{i}$ is considered a constant in all situations. Furthermore $e_{j}$ it will be assumed that the void is more than $10 \times$ is greatest linear dimension from any interface, such that the $\vec{\nabla} \lambda$ distribution within the void is eflectively uniform; $i$, , the existence of an interface does not perturb $\ddot{\nabla} \lambda$ in the void. This assumption implies that the concept of $h$ is valid and that (7) may be employed.

To ostablish the intluence of a heterogeneous system, we take as the reference valuc the induced charge $q$ which occurs in a homogeneous system for the same l' $(\vec{N})$ at the same woid location. Thus, with roforence to the induced charge $q_{n}$ of the heterogeneous system, we have

$$
\frac{q_{n}}{q}=\frac{-\vec{n} \cdot h_{n} \vec{\nabla} \lambda_{0 m}}{-\vec{i}+h \vec{\nabla} \lambda_{0}}
$$

whare $n=1,2$ depending in which dielectric medium the void is locatcd. For the homogeneous system $\lambda_{0}$ is independent of the specific diclectric. In the heterogeneous system, if the void is located in a dielectric of the same permittivity as that of the homogeneous reference dielectric, wo have equality of $h_{72}$ and $h_{\text {. }}$

Duc to the geometries under consideration, the dipole moment is dirceted either away from, or towards the coordinate origin. Hence this monent can be expressed as

$$
\ddot{\mu}=+\mu \vec{e}_{u}
$$

where $\vec{e}_{u}$ is a unit vector tangential with the coordinate $u$ where $u$ is the relevant coordinate for the geometry in question. IIence upon undertaking the vector operations, (12) is reduced to

$$
\frac{w_{m}}{q}=\frac{d \lambda_{b m} / d u}{d \lambda_{0} / d u}
$$

Providod the use of $h_{n}$ in (12) remains valid, (14) indicates that $q_{n} / a$ is independent of the votd geometry.

\subsection{PLANAR GEOMETRY}

Upon substituting the relewant $\lambda_{0}$ gradients (see Appendix) into (14), wo obtain for a woid in medium 1

$$
\frac{q_{1}}{q}=\frac{\varepsilon_{2} d}{\varepsilon_{1}(d-s)-1 \cdot \varepsilon_{2} s}
$$

and for a woid in medium 2

$$
\frac{q_{2}}{q}=\frac{\varepsilon_{1} d}{\varepsilon_{1}(d-s)+\varepsilon_{2} s}
$$

These expressions indicate that $q n / q$ is also independent of the void location in the specific medium, in so far as the $h$ validity reauirement is fulfilled. For $\varepsilon_{1}=\varepsilon_{g}$, the ratios in (15) and (16) reduce to unity.

The variations of $q_{\mathrm{n}} / q$ with $\varepsilon_{2} / \varepsilon_{1}$ are shown in Figure 2 for several values of $s / d$. From the Figurc it is seen that, in comparison to the homogeneous system, the induced charge of a heterogencous system is incrused when the void is located in the medium of losser permittivity. Likewise a decrease occurs for void locations in the medium of higher permittivity. When the void is in the lower dieletric (medium 1), the

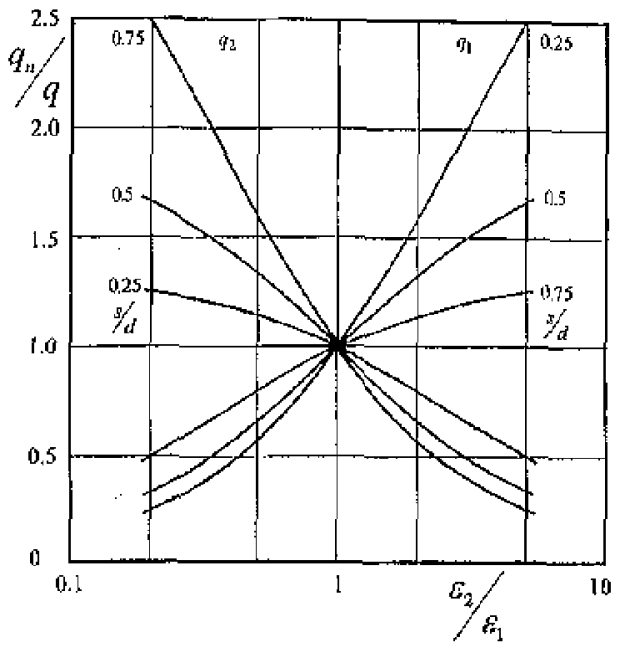

Figure 2. Wartation of $t_{n} / q$ wifh $\varepsilon_{2} / s_{1}$ for the planar genmatry and selected $s / d$ vilues.

nearer the dielectric interface is to the detecting electrode $(\lambda=1)$, the larger are the relative changes in these induced charges. The opposite behavior oceturs when the void is in the upper dielectric (medium 2); i.e. the further the interface is from the detecting olectrode the stronger is the effoct. $A$ s the value of $s / d$ represents the rolative thickness of medium 1 these results imply that, when the void is in the dielectric of smaller physical extent, the larger is the relative increase in afr. Owing to the geometrical symmetry of the planar system, the behavion of $q_{n}$ is symmetrical with respect to $s / d$ and $(1-s / d)$.

To simplify comparison with the other geometries the following convention will be adopted; vix the dielectric layer bounding the detecting clectrode has permittivity $\varepsilon_{\perp}$ and thickness $s$, while tho combined thickness of the two dielectic layers is $d$.

\subsection{CYLINDRICAL GEOMETRY}

Substitution of the relevant $\dot{\nabla} \lambda_{0}$ functions into (14) gives for a void in medium 1

and for a woid in medium 2

$$
\frac{q_{1}}{q}=\frac{\varepsilon_{2} \ln (b / a)}{\varepsilon_{1} \ln (b / c)+1 \cdot \varepsilon_{2} \ln (c / a)}
$$

$$
\frac{q_{2}}{q}==\frac{\varepsilon_{1} \ln (b / a)}{\varepsilon_{1} \ln (b / c)-1+\varepsilon_{2} \ln (c / a)}
$$

These expressions indicate that $q_{n} / q$ is also independent of the void location, so long as the previously discussed $h$, requirenent is fulfilled. Again, the ratios in (17) and (18) reduce to unity for $\varepsilon_{1}=-\varepsilon_{2}$.

When referring to cither the cylindrical or spherical geometries, we will use the product $H d d$ as a measure of the geometric non-uniformity. The parameter II is the mean curvature of the inner (detecting) electrode, while $d$ represents the total thickness of the dielectric insulation. Hence for the cylindrical geometry we have

$$
I I=\frac{1}{2 a}
$$

and

$$
d=b-a
$$


As the parameter $s$ represents the thickness of the dielectric bounding the detecting electrode, then for the cylindrical system we have

$$
s=c-u
$$

For a coaxial elcetrode system with a radii ratio of $e_{r} H d=0.859$, while for the planar electrode system, $I I d \equiv 0$.

The variations of $q_{n} / q$ with $\varepsilon_{2} / \varepsilon_{1}$ for the cylindrical geometry, $I r d=1$, ate illustrated in Figure 3 tor the same values of $s / d$ as in the planar case. The pattern of the variations is similar to that shown in Figure 2 for the planar geometiy, but now the simple symmetry of the pattern is absent; namely the increases and reductions in $q / q$ are loss, while the reverso behavion is exhibited by $q_{2} / q$. These results indicate that the geonetric nom-uniformity modifies the influence of the dielectric thickness. We proceed to examine further the intluence of the geometric parametos:

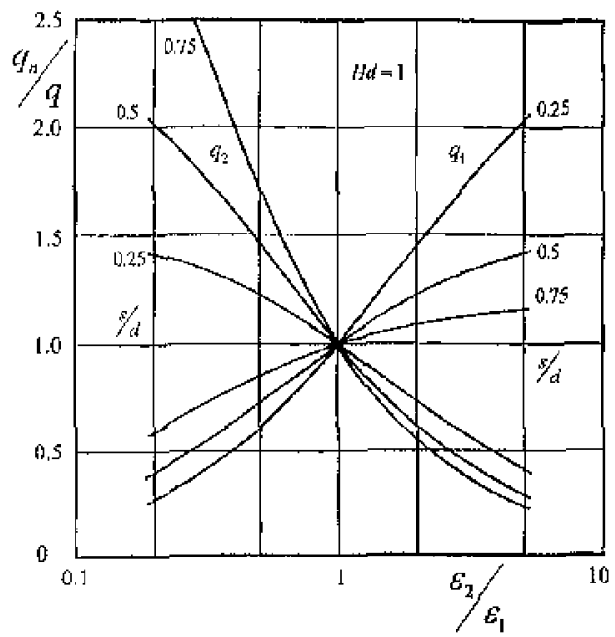

Figure 3, Variation of $q_{n} / q$ with $\varepsilon_{2} / s_{1}$, for the cylindrical geometry $(I / d=1$ ) and selectod $s / d$ values.

The effect of the non-unilomity of the geometry, i.e, the value of $H d$, upon the $a_{n} / q$ behavior is shown in Figure 4 . It is clear that increasing the value of $H d$ leads to a reduction in the increase/decrease of $a_{1} / q$ about the reference value of unity. This bohavior is more pronounced when the woid is in the dielectric of lower permittivity, i.e. for $q_{1} / q$ when $\varepsilon_{2} / \varepsilon_{1}>1$. The opposite trend is exhibited by $q_{2}$ in that increasing walues of $H d$ lead to an own larger increases/decreases in the $q_{2} / r$ behavior about unity.

\subsection{SPHERICAL GEOMETRY}

With respect to the spherical geoncty the mean curvature of the inner electrode is given by

$$
I T=: \frac{1}{A}
$$

while the total insulation thickness is now

$$
d=B-A
$$

and the thickness of the dielectric bounding the detecting electrode is

$$
s=C-A
$$

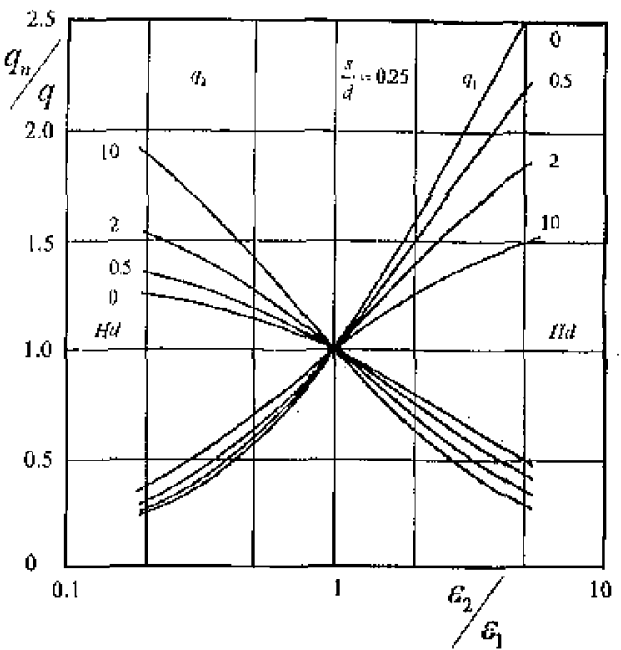

Figure 4. Varintion of $q_{n} / c_{1}$ with $\varepsilon_{2} / \epsilon_{3}$ for the cylindrical geometry and $s / d=0.25:$ Eiffect of $H t$.

Using (53), (54) and (55), the relevant $q_{n} / q$ expressions are derived from (14). However as the variations of $a_{n} / \mathrm{d}$ display fhe same behavior as these of the cylindrical geometry, it is not necssary to discuss the spherical geometry in detail.

In brict, it may be stated that, for $M d \leqslant 1$, the $g_{n} / q$ variation for the spherical geonctry are essentially identical to those exhibited by the cylindrical geometry. For $H d>1$, the $q_{1} / q$ variations begin to differ, in particular at the low $s / d$ values; i.e. the increases/reductions in $q_{1} / q$ for the spherical geonetry are smaller than those in the cylindrical case. The reverse belavior is observed with $q_{2} / q$.

\section{INDUCED CHARGE DUE TO $\delta \vec{l}$ IN HETEROGENEOUS DIELECTRIC SYSTEMS}

With respect to the component of the induced charge related to $\delta \vec{P}$, wo have upon combining (9) with (8)

$$
\frac{a_{p m}}{a_{1}}=\frac{-\vec{\mu} \cdot\left(h_{\mathrm{rl}} \vec{\nabla} \lambda_{b_{n}}-\vec{\nabla} \phi\right)}{-\vec{\mu} \cdot i_{t_{2}} \vec{\nabla} \lambda_{0_{n}}}
$$

where $n=1,2$ depending in which dielectric the woid is located. On performing the vector operations, (25) simplifies to give

$$
\frac{\eta_{m} m}{q_{n}}=1-\frac{d \phi / d u}{h_{n} d \lambda_{0 n} / d u}
$$

where $u$ is the relevant coordinate for the geometry under discussion. From (26) it is evident that the polarity of $q_{p_{n}} / q_{n}$ is dependent upon whether

$$
\frac{d d / d a t}{h_{n} d \lambda_{6 n} / d u} \leqslant 1
$$

That is the polarization component may woerse polatity. The polarity of the Poissonian induced charge, however, cannot exhibit such behavion; see (14).

Because (26) contains $/ h_{n}$, this implies that $q_{p n} / q_{n}$ is dependent upon the void geonelry. In this study, we will assume the void to be 
spherical, in which ase we have

$$
h=\frac{3 \varepsilon_{r}}{1+2 \varepsilon_{r}}
$$

where $\varepsilon_{T^{\prime}}$ is the relative permittivity of the diclectric containing the void.

With reference to $q_{n} / q_{r}$ ifs value when associated with the condition $q_{m n} / q_{n}=0$ can be deduced by using (26) together with the fact that for a homogeneous system $\lambda a$ and $\phi$ are synonymous. Thus, for zero polarization component, we obtain

$$
\left[\frac{q_{n}}{q}\right]_{0}=\frac{1}{i_{n n}}
$$

where the subscript 0 in (29) refers to this condition. For $\varepsilon_{r}>1$, we have $h_{p \mathrm{k}}>1$ and thus (29) implies that $0<\left(a_{m} / q\right)_{0}<1$.

\subsection{PLANAR HETEROGENEOUS DIELECTRIC GEOMETRY}

Employing (43), (44) and (45) we obfain for a void in medium 1

$$
\frac{q_{p \perp}}{q_{1}}=1-\frac{\varepsilon_{1}(d-s)+\varepsilon_{2} s}{\varepsilon_{2} h_{\perp} d}
$$

and for a roid in medium 2

$$
\frac{q_{12}}{q_{2}}=1-\frac{\varepsilon_{1}(d-s)+\varepsilon_{2} s}{\varepsilon_{1} h_{2} d}
$$

For $\varepsilon_{1}=\varepsilon_{2}$, equality of $h_{1}$ and $h_{2}$ exists and thus both (30) and (31) reduce to (11).

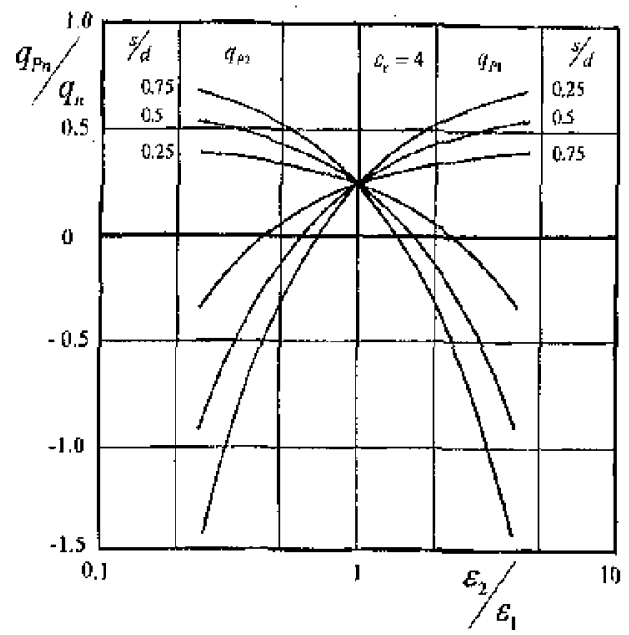

Figure 5. Variation of $q_{p n} / q_{n}$ with $\varepsilon_{2} / \varepsilon_{1}$ for the planar geometry and selected $s / d$ values $\left(\epsilon_{r}-4\right)$,

The variation of $q_{p m} / q_{\mathrm{r}}$ is shown in Figure 5 for $\varepsilon_{r}=4$ together with the pre-selected $s / d$ values. To ensure that the $q_{p n} / q_{n}$ calculations were undertaken for realistic $\varepsilon_{2} / \varepsilon_{1}$ values, the range of this parameter was restricted to

$$
\frac{1}{\varepsilon_{r}} \leqslant \frac{\varepsilon_{2}}{\varepsilon_{1}} \leqslant \frac{10}{\varepsilon_{r}}
$$

From Figure 5 it is observed that the behavior of $q_{p n} / q_{n}$ is similar to that shown in Figure 2 for $q_{n} / q ;$ i.e. $q_{p m} / q_{m}$ incteases when the woid is located in the dielectrie with the lesser permittivity. In marked contrast, the reduction whidt occurs when the void is in the medium of higher permittivity can be of such a degree that the polarity of $q_{p m}$ is reversed. The influence of $s / d$ witl respect to the magnitude of $q_{p n} / q_{i+}$ is similar to that observed with $a_{n} / q$; see Figure 2 .

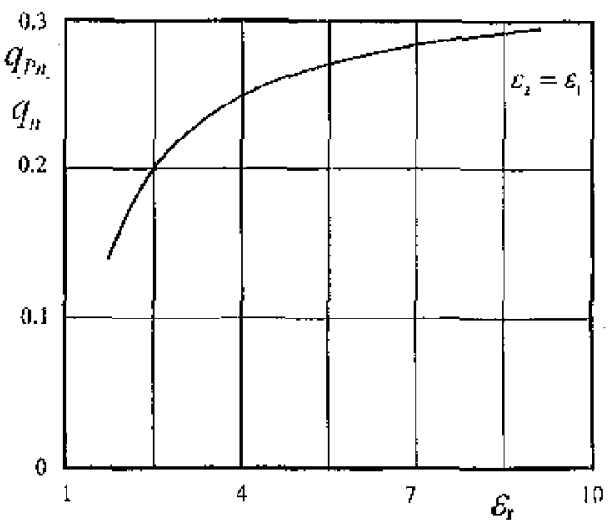

Figure 6. Variation of $q_{2 n} / q_{n}$ with $\varepsilon_{n}$, for $\varepsilon_{2} / \varepsilon_{1}=1$.

For $\varepsilon_{r}=6$, the variation of $q_{p n} / q_{n}$ with $\varepsilon_{2} / \varepsilon_{1}$ is similar to that shown in Figure 5 for $\varepsilon_{r}=4$. The magnitude of $q_{p r} / q_{n}$ is howower increased. This increase is proportional to the incruased value of $a_{p n} / a_{n}$ for $\varepsilon_{1}=\varepsilon_{2}$, i.e. to the crossover value observed in Figure 5 . For this condition, the variation of $\eta_{p n} / q_{n}$ with $\varepsilon_{n}$ is illustrated in Figure 6 . With the heterogencous system and $\varepsilon_{y}=2$, a similar variation to that shown in Figure 5 is found to be exhibited by $q_{p n} / q_{n}$. However, as can be understood from the reduced cross-over value for $\varepsilon_{r^{\prime}}=2$ shown in Figure 6 , there is a corresponding reduction in the $q_{j w} / q_{n}$ values with respect to those for $\varepsilon_{p}=4$,

\subsubsection{POLARITY REVERSAL OF POLARIZATION COMPONENT}

With respect to the polarity reversat of $q_{p} / q_{n}$, the value of $\varepsilon_{2} / \varepsilon_{1}$, corresponding to $q_{p 1} / q_{1}=0$ is obtained from $(30)$ ?

$$
\left[\frac{\varepsilon_{2}}{\varepsilon_{1}}\right]_{c}=\frac{d-s}{d_{l_{1}}-s}
$$

whilc from (31) the appropriate $\varepsilon_{1} / \varepsilon_{2}$ valtue for $q_{p 2} / q_{2}=0$ is

$$
\left[\frac{\varepsilon_{1}}{\varepsilon_{2}}\right]_{c}=\frac{s}{d\left(h_{2}-1\right)+s}
$$

The subscript ain (33) and (34) refers to the fact that the permittivity ratio has a critical value. As the polarity reversal occurs when the woid is in the dielectric of higher permittivity, this implies that the values of the ratios in (35) and (36) are $<1$, and that the pernittivity in the dcnominator represents the mediun containing the void:

The variations of the above permittivity ratios with $s / d$ for different $\varepsilon_{7}$ values ate shown in Figute 7 . For increasing values of $s / d$, it is clear that $\left(\varepsilon_{1} / \varepsilon_{2}\right)_{1}$ increases, while $\left(\varepsilon_{2} / \varepsilon_{1}\right)_{2}$ decreases correspondingly These results indicate that, for the dielectric containing the void, the smalier the physical dimensions are of this dielectric the more $\left(\varepsilon_{2} / \varepsilon_{1}\right)_{0}$ and $\left(\varepsilon_{1} / \varepsilon_{2}\right)_{i}$ tend to an upper limiting value. From (33) it is owident that as $s / d \rightarrow 0$

$$
\left[\frac{\varepsilon_{2}}{\varepsilon_{1}}\right]_{\mathfrak{c}} \rightarrow \frac{1}{h_{1}}
$$




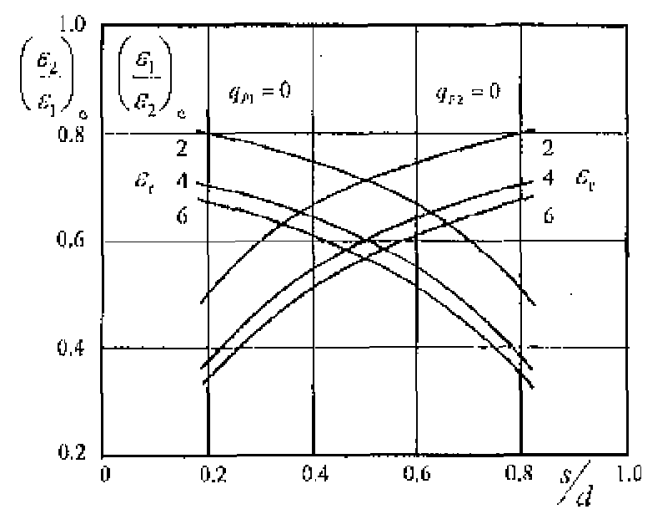

Figure 7. Variation of 'zero' permittivity ratios with $s / d$ for selected $\varepsilon_{\text {, }}$ vilues.

while from (34) we find that as $s / d \rightarrow 1$

$$
\left[\frac{\varepsilon_{1}}{\varepsilon_{2}}\right]_{c} \rightarrow \frac{1}{h_{2}}
$$

As the permittivity atios for practical situations lie in the range 0.4 to 0.7 , the possibility for polarity reversul of $q_{j n} / a_{n}$ is real. Planar systems are frequently employed in laboratory studies.

\subsection{CYLINDRICAL HETEROGENEOUS DIELECTRIC GEOMETRY}

Using (48), (49) and (50) we obtain for a void in medium 1

$$
\frac{q_{p 1}}{a_{1}}=1-\frac{\varepsilon_{1} \ln (b / c)+\varepsilon_{2} \ln (c / a)}{\varepsilon_{2} h_{1} \ln (b / a)}
$$

and for the woid in mediun 2

$$
\frac{q_{p 2}}{a_{2}}=1-\frac{\varepsilon_{1}}{-\frac{\ln (b / c)+\varepsilon_{2} \ln (c / a)}{\varepsilon_{1} h_{2} \ln (b / a)}}
$$

As before, for $\varepsilon_{1}=\varepsilon_{2}$, we obtain equality of $h_{1}$ and $h_{2}$ and hence (37) and (38) reduce to (11).

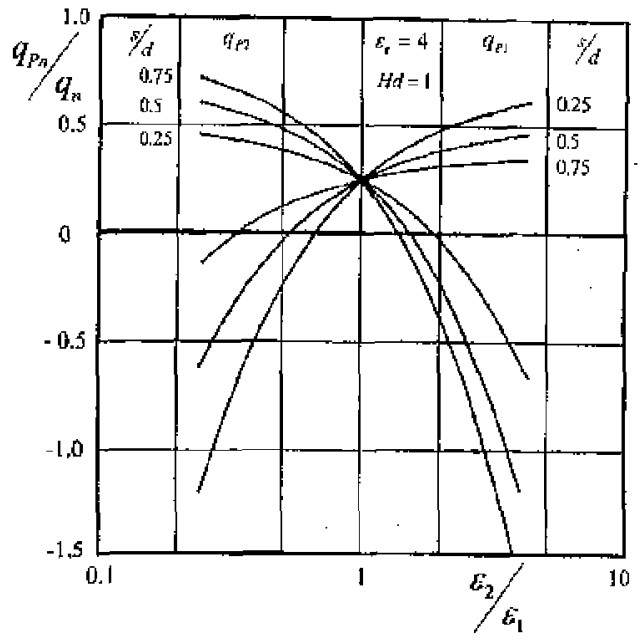

Figure 8. Wartation of $q_{1 r^{\prime}} / q_{\mathrm{n}}$ with $\varepsilon_{2} / \varepsilon_{1}$ for the cylindrical geometry $(H d=1)$ and sclected $s / d$ values $\left(\epsilon_{r}=4\right)$.
The variations of $q_{p n} / q_{n}$ with $\varepsilon_{2} / \varepsilon_{1}$ for the cylindrical geometry, $I T d=1$, are shown in Figure 8 . It is clear that these variations are similar to those of the planar goometry, cf. Figure 8 with Higute 5 . The primary difference is the absence of symmetry in the case of the cylindrical geometry. This results from the increases/reductions in $q_{p} 1 / q_{1}$ being less, while those for $q_{p 2} / q_{2}$ are larges.

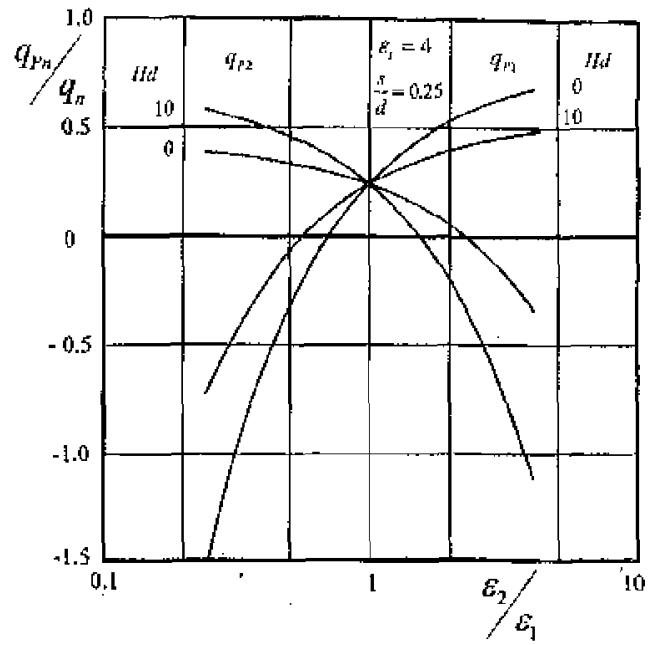

Figure 9 . Wiriation of $q_{p r z} / q_{\mathrm{r}}$ with $\varepsilon_{2} / \varepsilon_{1}$ for the cylindrical geonetry and $s / d=0.25$ : Effect of $H d\left(\varepsilon_{\tau^{\prime}}=4\right)$.

The influence of system geometry is fully illustrated in Figure 9 with the variation of $q_{\mathrm{m}} / \mathrm{q}_{\mathrm{m}}$ for $I I d=0$ and $H d=: 10$. In comparison with Figure 8 it is evident that, for the parameter ranges considered, $a_{p n} / q_{n}$ is more sensitive to $s / d$ than to $H d$.

The effect of $\varepsilon_{\text {: }}$ upon $q_{p m} / q_{n}$ for the coaxial geomotry is similar to that as discussed proviously for the planar geometry, with the addition of the asymmetry shown in Figure 8 .

\subsection{1' POLARITY REVERSAL OF POLARIZATION COMPONENT}

For the cylindrical geonetry the value of $\varepsilon_{2} / \varepsilon_{1}$ corresponting to $q_{21} / q_{1}=0$ is given by

$$
\left[\frac{E_{2}}{\varepsilon_{1}}\right]_{c}=\frac{\ln (b / c)}{h_{1} \ln (b / a)-\ln (c / a)}
$$

while the appropriate $\varepsilon_{1} / \varepsilon_{2}$ value for $q_{y 2} / q_{2}=0$ is

$$
\left[\frac{\varepsilon_{1}}{\varepsilon_{22}}\right]_{0}=\frac{\ln (c / a)}{h_{2}} \frac{\ln (b / a)-\ln (b / c)}{\ln (d)}
$$

The variation of these permittivity ratios with $I / d$ for the selected values of $s / d$ are illustrated in Figure 10 for $\varepsilon_{v}=1$. Essentially the same behavior is exhibicd for $\varepsilon_{r}=2$ and $\varepsilon_{r}=6$. For $I / d$ values of practical interest for solid insulation $(H d<2$ ), the permitrivily ratios generally lie in the range 0.4 to 0.7. Such values imply that the polarity reversal of $q_{r}$ is quite possible in practical systems.

\subsection{SPHERICAL HETEROGENEOUS DIELECTRIC GEOMETRY}

By employing (53), (54) and (55), we obtain from (26) the relevant $a_{p m} / a_{n}$ expressions for this geometry. From thase expressions it is 


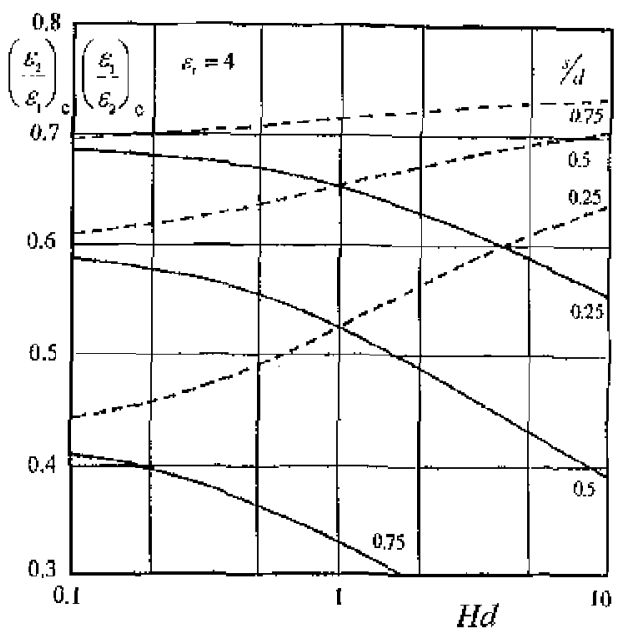

Figure 10. Wartation of "zero' permittivity watios with $H d$ for the cylindrical geometry and selected $s / d$ values $\left(\varepsilon_{r}=4\right)$. - $\left(\varepsilon_{2} / \varepsilon_{1}\right)_{e}$ for $q_{p 1}:-0_{1}--:\left(\varepsilon_{1} / \varepsilon_{2}\right)_{c}$ for $q_{p 2}=0$.

readily demonstrated that the variations of $q_{p n} / q_{n}$ exhibit the same characteristics as were discussed previously with respect to the corresponding $q_{n} / q$; i.e. the spherical $q_{p n} / q_{n}$ behavior is similar to that of the cylindrical geometiy. Conscquently it is unnecessary to discuss in detail the $q_{p m} / q_{n}$ results for the sphorical geomelry.

\section{DISCUSSION}

\subsection{GENERAL REMARKS}

The Poissonian induced charge $q$ has boen shown mathematically to consist of two components [2]. One component $q_{t i}$ is related directly to the space charge created in the void by the PD. The other component $q_{p}$ is associated with the change in the polarization of the solid dielectric arising from the action of the electric field produced by the void space charge.

The ability to decompose mathematically the Poissontan induced charge into these two components rela tes to the use of the $\lambda$ and $b$ functions to evaluate induced charge. The former accounts for the dielectric polarization implicitly, while the latter function does so explicitly [2].

The combined use of these functions allows quantitative relations for $g_{j}$ to be deduced, viz. (6) or (9). Each of these expressions contains throe independent vectors, and thus for heterogeneous bulk diclectric systems the behavior of $q_{p}$ is complex. Consequently to obtain insight into this behavior, the present study has been restricted to situations in which the three vectors are parallel. For heterogeneous bulk dielectric systems such a requirement can be fulfilled by simple goometries, e.g. planar; cylindrical and spherical electrode systems. The planar geometry $(I / d=0)$ enables the basic foatures of a heterngeneous system to be deduced, but is limited in that the effects of system non-uniformity annot be addressed. Howcver this aspect can be fully considered through cylindrical and spherical systems, as such gometries can be used to gencrate a wide variation in non-uniformitys eg. $0<H d<10$.

\subsection{INFLUENCE OF HETEROGENEOUS BULK DIELECTRIC SYSTEMS UPON INDUCED CHARGE}

By omparing the l'oissonian induced charge associated with a PD in a void located within a heterogencous system with that of a corresponding homogeneous system, it has been possible to illustrate the influence of the former. As the same void geonetry is considered in both situations, the comparisot is made independent of the void goonetry,

Under the assumption of a constant pD (i.e. constant dipole moment), it is demonstrated that the magnitude of the Poissonian induced charge can vay significantly, e. $8.50 \%$, For the present two dielectric system, this behavior is seen to be dependent upon the ratio of the permittivities and within which diclectric the void is located.

The induced charge magnitude is firther influctced by the degree of non-uniformity of the system geonetry; i.e. symmetrical induced charge variations ( $I A d=0$ ), vs. asymmetrical variations $(I I d>0)$. The larger the value of $H d \mathrm{i}$ s, the higher is the degree of asymmetry. For the cylindrical and spherical geometrics essentially identical induced charge variations are produced for $H d<1$.

The asymmetry in the induced charge variation for non-uniform. geometries ( $H d>0$ ) could be interpreted as indicating that, depending on woid location, there was a preforential detecting electrode, i.e. the outer instead of the inmer electrode. With respect to woid $\mathrm{PD}$, the induced charge is proportional to $\vec{\nabla} \lambda_{0}$ not $\lambda_{0}$; see (8). For the present 2 electrode geometries $\vec{\nabla} \lambda_{0}$ is independent of which clectrode is denoted the detecting electrode, and thus no preferential electrode exists.

The magnitude of the induced chargo is also influenced by the physical dimensions of the dielectric containing the void; wiz the snaller the dielectric is in extent, the larger is the change in magnitude. This aspect is clearly illustrated by the symmetry in the planar resuls.

\subsection{INFLUENCE OF CHANGES IN BULK DIELECTRIC POLARIZATION UPON INDUCED CHARGE}

The ratio of the polarization component to the Poissonian induced charge was cmployed to illustrate the manner in which a heterogeneous dielectric system affected the induced charge associated with changes in the polarization of the bulk diclectric. The polarization component increased when the woid was located in a dielectric of lesser permittivity while a decrease occutred when located in a dielectric of higher permittivity. This reduction was observed to be so marked that the polarity of the polarization component was reversed.

For the $q_{m n} / q_{n}=0$ condition, the permittivity ratios for practical situations lic in the range 0.4 to 0.7 . Hence the chance of this condition arising in practice is high, particularly if, for $I J>0$, the inner dielectric is selectid to have the higher permittivity. This is common practioc to reduce operating stress levels.

The system goometry and the dielectric dimensions also influence the $q_{n+m} / q_{n}$ ratio. These variations reflect those encountered for $q_{n} / q$ for the heterogeneous/homogeneous systems. 
In our analytical approach, it should be noted that the $\lambda$ and $\phi$ functions are employed to covaluate directly the charge induced on the detocting electrode. This charge is an infegral property of the field produced by the PD. Hence the evaluation procedure cannot provide detailed information on the point properties of the field created by the po. Thus although the polarity reversal behavior of the polarization component is revealed, an in-depth understanding of the phenomenon will necessitate a full field solution. Such a solution is outside the scope of the prosont study.

\subsection{PRACTICAL ASPECTS}

To fully exploit the present theoretical study, the location of the $\mathrm{PD}$ was restricted only with distance from an interface: see Section 4 . This freedom of location does not exist in practical situations, as a minimum clectric field strength is necessary to create the conditions for l'L development. Such conditions are invariably fulfilled only in the vicinity of the conductor with greatest curvature. For the cylindrical and spherical geonetries, this implies that with the P'D void located in the inner dielectric (medium 1) the results of the theoretical study relate dircotly to situations readily encountered in practice.

It has been shown previously for a homogeneous dielcetric system that a quantitative evaluation of a $\mathrm{SD}$ transient requires a knowledgc of the void location, geometry and dimensions, void gas pressure and composition and the woid orientation with reference to the applied ficld $\left[4_{f}\right.$ 5]. For heterogenenus dielectric systems, the present study indicates that the situation becomes even more conplox.

In the practical world, it is highly unlikely that all the nocessary information required to undertake a quantitative evaluation of plu masutements would be arailable. Consequently such measurements can only be handled in terms of patton recognition. However to undertake meaningful comparisons with previous data, such that tends become identifiable, the mensurements should be restricted to similar cquipment produced by the same manufacture: In this way the many parameters mentioned above would remain essentially und anged.

Shout however a manufacturer change the insulation materials employed in the production process, then this could have a significant of-

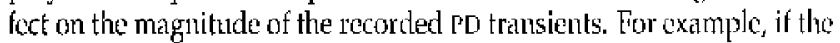
permittity ratio were altered, then, for the same $\mathrm{PD}$, the recorded transient would show cither an increase or a decronse. This in turn could lead to an incorrect assessment of the status of the insulation, particulatly if the criterion for assosment were based on a fixed $\mathrm{pC}$ level of activity. Consequently any change in the insulation materials should be accompanied by the acquisition of a now reference set of l'D data.

\section{CONCLUSIONS}

W E have examined the Poissonian induced charge in terms of two components; one due directly to the void space charge, and one due to changes in the dielectric polarization brought about by the former.

For a haterogenums bulk dielectric systen, it is demonstrated that changes in the dielectric polarization can significantly influence the magnitude of the Poissonian induced chatget e.g. a promounced roversol in the polarity of the polarization component is possible. This behavior is not observed in a homogeneous diclectric system. For a two dielectric system, the scale of this influence is dependent primarily upon the ratio of the diclectric permitivities and within which diclectric the woid is located. 'l'he physical dimensions of the different diclectrics and the goometry of the dielectric system cxert a similar, although losser effect upon the magnitude of the induced charge.

On the basis of these observations, we conclude that a correct quantitative interpretation of Je tratsients in tornes of woid discharge phenomena cannot be adieved for practical, heterogeneous bulk diclectric systems. Moreorer fixed pC level assessment should be treated with extrene catution.

\section{APPENDIX}

\subsection{PLANAR ELECTRODE GEOMETRY}

Consider a two layor diolectric bounded by planar electrodes. If in recangular coordinates, the electrodes are represented by $z=0$ and $z=d_{r}$ then the dielectric inlerface is taken as $z=s$, with $s<d$. The permittivity of the upper dielectric is $\varepsilon_{2}$ for which $s \leqslant z \leqslant d$, while that of the lower is $\varepsilon_{1}$, lor which $0 \leqslant z \leqslant s$.

If the lower electrode is used as the detecting clectrode, then the boundary conditions for the $\lambda_{0}$ function are $\lambda_{0}-1$ for $z=0$ and $\lambda_{0}=0$ for $z=d$. Hence the $\lambda_{0}$ functions of the two media are given by

for $0 \leqslant z \leqslant 4$, and

$$
\lambda_{01}=\frac{\varepsilon_{1}(d-s) \cdot \mid \cdot \varepsilon_{2}(s \cdots z)}{\varepsilon_{3}(d-s)+\varepsilon_{2} s}
$$

$$
\lambda_{02}=-\frac{\varepsilon_{1}(d-z)}{\varepsilon_{1}(d-s)+\varepsilon_{2} s}
$$

for $s \leqslant z \leqslant d$, where the $\lambda_{0}$ subscripts, 1 and 2 , refor to the lower and upper regions, respectively. On differentiating with respoct to $z$, we obtain the relevant expressions for the associated $\lambda_{0}$ gradients

$$
\vec{\nabla} \lambda_{01}=\frac{-\varepsilon_{2} \vec{c}_{z}}{\varepsilon_{1}(d \cdots s)-\varepsilon_{2}, 5}
$$

and

$$
\vec{\nabla} \lambda_{\alpha_{2}}=-\frac{-\varepsilon_{1} \vec{e}_{z}}{\varepsilon_{1}(d-s)+\varepsilon_{2 y}}
$$

where $z$ is a unit vector in the positive $z$ direction. For a homogeneous medium, $\lambda_{0}=\phi$ and thus for a planar system we have

$$
\vec{\nabla} \lambda_{0}-\vec{\nabla} \phi=-\frac{\vec{r}_{z}}{d}
$$

Both (43) and (44) reduce to this expression for $\varepsilon_{1}=\varepsilon_{2}$.

\subsection{CYLINDRICAL ELECTRODE GEOMETFY}

We consider a coxial cylindrical geometry with a two layer dielectric. If, in cylintrical coordinates, the inner electrode is represented by the surface $r=a$, while $r=b$ is the inner surface of the outer elec trode, then $r=$ : e represents the diclectric interface with $a<a<b$. The permittivity of the inner dielectric is $\varepsilon_{\text {, }}$ for which $a \leqslant r \leqslant c$, while that of the outer is $\tau_{2}$, for which $c \leqslant \gamma \leqslant b$. 
If the complcte inner electrode is used to detect the r'D transient, then the boundary condifions for the $\lambda_{0}$ function are $\lambda_{0}=1$ for $r=a$ and $\lambda_{0}=0$ tor $r=b$. Hence the $\lambda_{0}$ functions of the two media are given by

$$
\lambda_{01}=\frac{\varepsilon_{1} \ln (b / c)+\varepsilon_{2} \ln (c / r)}{\varepsilon_{1} \ln (b / c)+\varepsilon_{2} \ln (c / a)}
$$

for $u \leqslant r \leqslant c$, and

$$
\lambda_{02}=\frac{\varepsilon_{1} \ln (b / r)}{s_{1} \ln (b / c)+\varepsilon_{2} \ln (c / a)}
$$

for $c \leqslant r \leqslant b$. The $\lambda_{0}$ subscripts, 1 and 2 , refer now to the inner and outer regions, respectively.

Upon differentiating with respect to $r$, we obtain the relevant $c x$ pressions for the associated $\lambda_{0}$ gradients

and

$$
\vec{\nabla} \lambda_{01}=\frac{-\left(\varepsilon_{2} / r\right) \vec{c}_{r}}{\varepsilon_{1} \ln (b / c)+\varepsilon_{2} \ln (c / a)}
$$

$$
\ddot{\nabla} \lambda_{02}=\frac{-\left(\varepsilon_{1} / r\right) \vec{c}_{r}}{\varepsilon_{1} \ln (b / c)+\varepsilon_{2} \ln (c / a)}
$$

where $\vec{e}_{r}$ is a unit vector perpendicula: to the axis of the coaxial system and directed away from the inner electrode.

For a homogeneous medium, $\lambda_{0}==\phi$ and thus for a coaxial system we have

$$
\vec{\nabla} \lambda_{0}=\vec{\nabla} \phi=\frac{-\vec{e}_{r}}{v \ln (b / a)}
$$

Both (48) and (49) reduce to this expression for $\varepsilon_{1}=\mathrm{Ea}$.

\subsection{SPHERICAL ELECTRODE GEOMETRY}

Consider a concentric spherical geometry with a two layer dielectric. If, in spherical coordinates, the inner electrode is represented by the surface $R=A$, while $R=B$ is the inner surface of the outer aloctrode, then $R=C$ represents the dielectric interface with $A<O<B$. The permittivity of the inner dielectric is $\varepsilon_{1}$ for which $A \leqslant R \leqslant C$, while that of the outer is $\varepsilon_{2}$, for which $C \leqslant R \leqslant B$.

If the complete inner electrode is uscd to detect the PD transient, then the boundary conditions for the $\lambda_{0}$ function are $\lambda_{0}=1$ for $\pi=A$ and $\lambda_{0}=0$ for $R=B$. Hence the $\lambda_{0}$ functions of the two media are given by

$$
\lambda_{01}=\frac{\varepsilon_{1}(1-C / B)+\varepsilon_{2}(C / R-1)}{\varepsilon_{1}(1-C / B)+\varepsilon_{2}(C / A \cdots 1)}
$$

for $A \leqslant R \leqslant C$, and

$$
\lambda_{(22}=\frac{\varepsilon_{1}(C / R-C / B)}{\varepsilon_{1}(1-C / B)+\varepsilon_{2}(C / A-1)}
$$

for $C \leqslant R \leqslant R$. The $\lambda_{0}$ subscripts, 1 and 2 , refor to the imner and outer regions.

Differentiating with respect to $R$ leads to the relevant expressions. for the corresponding $\lambda_{0}$ gradients

and

$$
\begin{aligned}
\vec{\nabla} \lambda_{01} & =-\frac{1}{R} \frac{\varepsilon_{2}\left(C / R_{1}\right) e_{R}}{\varepsilon_{1}(1-C / B)+\varepsilon_{2}(C / A-1)} \\
\vec{\nabla} \lambda_{n_{2}} & =-\frac{1}{R} \frac{\varepsilon_{1}(C / R) \vec{e}_{R}}{\varepsilon_{1}(1-C / B)+\varepsilon_{2}(C / A-1)}
\end{aligned}
$$

where $\vec{c}_{R}$ is a unit vector tangential with the radial coordinate and directed away from the inner clectrode.

For a homogeneous medium, $\lambda_{0}=\phi$ and thus for a spherical system we have

$$
\ddot{\nabla} \lambda_{0}=\vec{\nabla} \dot{\phi}=-\frac{1}{R} \frac{(B / R) \vec{e}_{R}}{(B / A)-1}
$$

For $\varepsilon_{1}=\varepsilon_{2}$, both (53) and (54) reduce to this expression.

\section{ACKNOWLEDGMENT}

The unigue contribution of the late A. Pedersen to the initial stages of this study is gratefully acknowledged.

\section{REFERENCES}

[1] A. Pedersen, G. C. Crichton and I. W. McAllister, "The Theory and Weasurement of Fartial Discharge Transicnts", ITEE Trans. Elect. Insul., Val, 26, pp. 467-497, 1991.

[2] A. Federsen, G, C.Crichton and I. W. Mcdllister, ${ }^{2}$ The Functional Rodation Betwen Partial Discharges and Ind uced Charge", IEFF, Trems. Diel. \& Elect. Insul, val. 2, pp. $535-543,1985$.

[3] A. Pedersen, G, C. Crichton and I. W. McAlister, "Tartial Discharge Delection: Theoretical and P'ractical Aspects", lEF Proc, - Science, Weasurement and Technology, vol. 142 , pp, 20-36, 1995.

[4] G. C. Crichton, P. W. Karleson and A. Pedergen, "Partal Discharges in Fllipsoidal and Sphetridal Wids", IEEE Träns, Flect. Inst.., Vhl. 24, pp. 335-342, 1989.

[5] 1. W. MaAllister "Tartial Discharges in Spheroidal Voids; Voic Orientation". JEEE T'rans. Diel. Elect. Iñull., vol. 4, pp. 456-461, 1997 ,

This paper is based on presentations given at fhe Conference on Electrical Insulation and Didectric Phenomena, Virginia Beach, VA, 1995 and San Irancisco, CA, 1096 and at the 1997 Volta Colioquim on Partial Discharge Moasurements, Como, Italy, 1997 .

Manuscript was received on 14 january 1908 . 